\title{
LIM-LIG at SemEval-2017 Task1: Enhancing the Semantic Similarity for Arabic Sentences with Vectors Weighting
}

\author{
El Moatez Billah Nagoudi \\ Laboratoire d'Informatique \\ et de Mathématiques \\ LIM \\ Université Amar Telidji \\ de Laghouat, Algérie \\ e.nagoudielagh-univ.dz
}

\author{
Jérémy Ferrero \\ Compilatio \\ 276 rue du Mont Blanc \\ 74540 Saint-Félix, France \\ LIG-GETALP \\ Univ. Grenoble Alpes, France \\ jeremy.ferrerodimag. fr
}

\author{
Didier Schwab \\ LIG-GETALP \\ Univ. Grenoble Alpes \\ France \\ didier.schwabeimag. fr
}

\begin{abstract}
This article describes our proposed system named LIM-LIG. This system is designed for SemEval 2017 Task1: Semantic Textual Similarity (Track1). LIM-LIG proposes an innovative enhancement to word embedding-based model devoted to measure the semantic similarity in Arabic sentences. The main idea is to exploit the word representations as vectors in a multidimensional space to capture the semantic and syntactic properties of words. IDF weighting and Part-of-Speech tagging are applied on the examined sentences to support the identification of words that are highly descriptive in each sentence. LIM-LIG system achieves a Pearsons correlation of 0.74633 , ranking 2 nd among all participants in the Arabic monolingual pairs STS task organized within the SemEval 2017 evaluation campaign.
\end{abstract}

\section{Introduction}

Semantic Textual Similarity (STS) is an important task in several application fields, such as information retrieval, machine translation, plagiarism detection and others. STS measures the degree of similarity between the meanings of two text sequences (Agirre et al., 2015). Since SemEval 2013, STS has been one of the official shared tasks.

This is the first year in which SemEval has organized an Arabic monolingual pairs STS. The challenge in this task lies in the interpretation of the semantic similarity of two given Arabic sentences, with a continuous valued score ranging from 0 to 5. The Arabic STS measurement could be very useful for several areas, including: disguised plagiarism detection, word-sense disambiguation, la- tent semantic analysis (LSA) or paraphrase identification. A very important advantage of SemEval evaluation campaign, is enabling the evaluation of several different systems on a common datasets. Which makes it possible to produce a novel annotated datasets that can be used in future NLP research.

In this article we present our LIM-LIG system devoted to enhancing the semantic similarity between Arabic sentences. In STS task (Arabic monolingual pairs) SemEval 2017, the LIM-LIG system propose three methods to measure this similarity: No weighting, IDF weighting and Partof-speech weighting Method. The best submitted method (Part-of-speech weighting) achieves a Pearsons correlation of 0.7463 , ranking 2 nd in the Arabic monolingual STS task. In addition, we have proposed another method (after the competition) named Mixed method, with this method, the correlation rate reached 0.7667 , which represent the best score among the different submitted methods involved in the Arabic monolingual STS task.

\section{Word Embedding Models}

In the literature, several techniques are proposed to build word-embedding model.

For instance, Collobert and Weston (2008) have proposed a unified system based on a deep neural network architecture. Their word embedding model is stored in a matrix $M \in R^{d *|D|}$, where $D$ is a dictionary of all unique words in the training data, and each word is embedded into a d-dimensional vector. Mnih and Hinton (2009) have proposed the Hierarchical Log-Bilinear Model (HLBL). The HLBL Model concatenates the $n-1$ first embedding words $\left(w_{1} . . w_{n-1}\right)$ and learns a neural linear model to predicate the last word $w_{n}$.

Mikolov et al. (2013a, 2013b) have proposed 
two other approaches to build a words representations in vector space. The first one named the continuous bag of word model CBOW (Mikolov et al., 2013a), predicts a pivot word according to the context by using a window of contextual words around it. Given a sequence of words $S=w_{1}, w_{2}, \ldots, w_{i}$, the CBOW model learns to predict all words $w_{k}$ from their surrounding words $\left(w_{k-l}, \ldots, w_{k-1}, w_{k+1}, \ldots, w_{k+l}\right)$. The second model SKIP-G, predicts surrounding words of the current pivot word $w_{k}$ (Mikolov et al., 2013b).

Pennington et al.(2014) proposed a Global Vectors (GloVe) to build a words representations model, GloVe uses the global statistics of wordword co-occurrence to calculate the probability of word $w_{i}$ to appear in the context of another word $w_{j}$, this probability $P(i / j)$ represents the relationship between words.

\section{System Description}

\subsection{Model Used}

In Mikolov et al. (2013a), all the methods (Collobert and Weston, 2008), (Turian et al., 2010), (Mnih and Hinton, 2009), (Mikolov et al., 2013c) have been evaluated and compared, and they show that CBOW and SKIP-G are significantly faster to train with better accuracy compared to these techniques. For this reason, we have used the CBOW word representations for Arabic model ${ }^{1}$ proposed by Zahran et al. (2015). To train this model, they have used a large collection from different sources counting more than 5.8 billion words including: Arabic Wikipedia (WikiAr, 2006), BBC and CNN Arabic corpus (Saad and Ashour, 2010), Open parallel corpus (Tiedemann, 2012), Arabase Corpus (Raafat et al., 2013), Osac corpus (Saad and Ashour, 2010), MultiUN corpus (Chen and Eisele, 2012), KSU corpus (ksucorpus, 2012), Meedan Arabic corpus (Meedan, 2012) and other (see Zahran et al. 2015).

\subsection{Words Similarity}

We used CBOW model in order to identify the near matches between two words $w_{i}$ and $w_{j}$. The similarity between $w_{i}$ and $w_{j}$ is obtained by comparing their vector representations $v_{i}$ and $v_{j}$ respectively. The similarity between $v_{i}$ and $v_{j}$ can be evaluated using the cosine similarity, euclidean distance, manhattan distance or any other similarity measure functions. For example, let "جامعة"

\footnotetext{
${ }^{1}$ https://sites.google.com/site/mohazahran/data
}

(university), "المساء" (evening) and "لملية" (faculty) be three words. The similarity between them is measured by computing the cosine similarity between their vectors as follows:

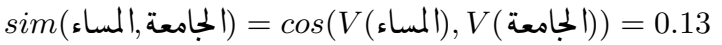

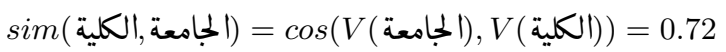

That means that, the words "لكلية" (faculty) and "الجامعة" (university) are semantically closer than "الماء" (evening) and "الماءعة" (university).

\subsection{Sentences similarity}

Let $S_{1}=w_{1}, w_{2}, \ldots, w_{i}$ and $S_{2}=w_{1}^{\prime}, w_{2}^{\prime}, \ldots, w_{j}^{\prime}$ be two sentences, their words vectors representations are $\left(v_{1}, v_{2}, \ldots, v_{i}\right)$ and $\left(v_{1}^{\prime}, v_{2}^{\prime}, \ldots, v_{j}^{\prime}\right)$ respectively. There exist several ways to compare two sentences. For this purpose, we have used four methods to measure the similarity between sentences. Figure 1 illustrates an overview of the procedure for computing the similarity between two candidate sentences in our system.

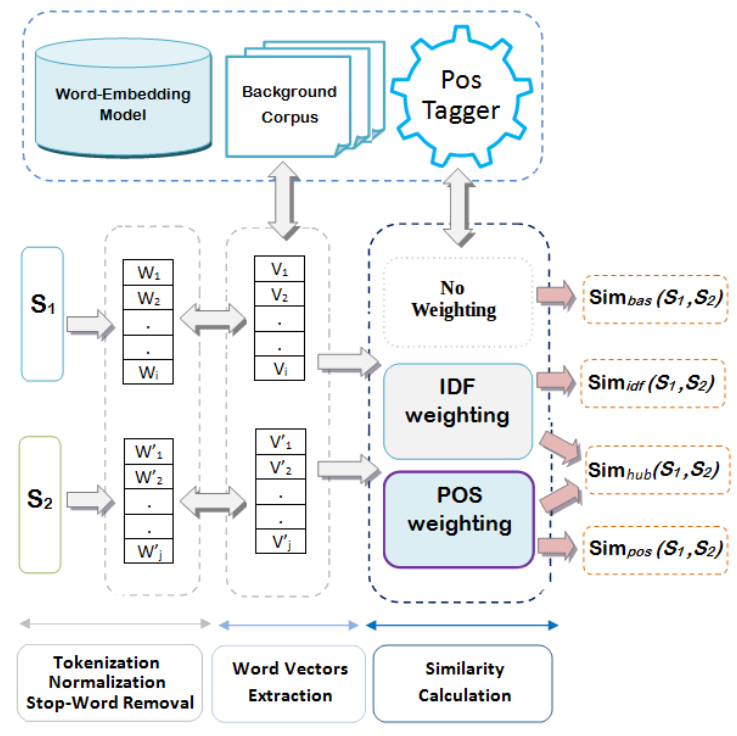

Figure 1: Architecture of the proposed system.

In the following, we explain our proposed methods to compute the semantic similarity among sentences.

\subsubsection{No Weighting Method}

A simple way to compare two sentences, is to sum their words vectors. In addition, this method can be applied to any size of sentences. The similarity between $S_{1}$ and $S_{2}$ is obtained by calculating the cosine similarity between $V_{1}$ and $V_{2}$, where:

$$
\left\{\begin{array}{l}
V_{1}=\sum_{k=1}^{i} v_{k} \\
V_{2}=\sum_{k=1}^{j} v_{k}^{\prime}
\end{array}\right.
$$


For example, let $S_{1}$ and $S_{2}$ be two sentences:

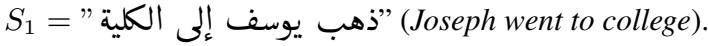

$S_{2}=$ "يوسف يمضى مسرعا للجامعة الكية "Joseph goes quickly to university).

The similarity between $S_{1}$ and $S_{2}$ is obtained as follows:

\section{Step 1: Sum of the word vectors}

$$
\begin{aligned}
& V_{1}=V(\text { (الكلية) }
\end{aligned}
$$

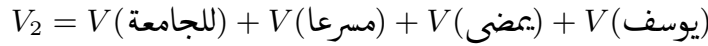

\section{Step 2: Calculate the similarity}

The similarity between $S_{1}$ and $S_{2}$ is obtained by calculating the cosine similarity between $V_{1}$ and $V_{2}: \operatorname{sim}\left(S_{1}, S_{2}\right)=\cos \left(V_{1}, V_{2}\right)=0.71$

In order to improve the similarity results, we have used two weighting functions based on the Inverse Document Frequency IDF (Salton and Buckley, 1988) and the Part-Of-Speech tagging (POS tagging) (Schwab, 2005) (Lioma and Blanco, 2009).

\subsubsection{IDF Weighting Method}

In this variant, the Inverse Document Frequency IDF concept is used to produce a composite weight for each word in each sentence. The idf weight serves as a measure of how much information the word provides, that is, whether the term that occurs infrequently is good for discriminating between documents (in our case sentences). This technique uses a large collection of document (background corpus), generally the same genre as the input corpus that is to be semantically verified. In order to compute the idf weight for each word, we have used the BBC and CNN Arabic corpus $^{2}$ (Saad and Ashour, 2010) as a background corpus. In fact, the $i d f$ of each word is determined by using the formula: $i d f(w)=\log \left(\frac{S}{W S}\right)$, where $S$ is the total number of sentences in the corpus and $W S$ is the number of sentences containing the word $w$. The similarity between $S_{1}$ and $S_{2}$ is obtained by calculating the cosine similarity between $V_{1}$ and $V_{2}, \cos \left(V_{1}, V_{2}\right)$ where:

$$
\left\{\begin{array}{l}
V_{1}=\sum_{k=1}^{i} i d f\left(w_{k}\right) * v_{k} \\
V_{2}=\sum_{k=1}^{j} i d f\left(w_{k}^{\prime}\right) * v_{k}^{\prime}
\end{array}\right.
$$

and $i d f\left(w_{k}\right)$ is the weight of the word $w_{k}$ in the background corpus.

Example: let us continue with the sentences of the previous example, and suppose that IDF weights of their words are:

\footnotetext{
${ }^{2}$ https://sourceforge.net/projects/ar-text-mining/files

\begin{tabular}{|c|c|c|c|c|c|}
\hline ذهب & يوسف & الكلية & يمضى & مسرعا & بامعة \\
\hline 0.27 & 0.37 & 0.31 & 0.29 & 0.22 & 0.34 \\
\hline
\end{tabular}
/Arabic-Corpora/
}

Step 1: Sum of vectors with IDF weights

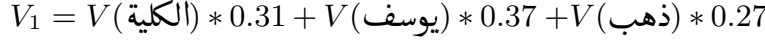

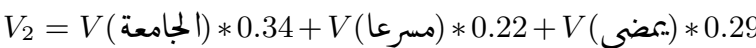

$$
+V(\text { ) }) 0.37
$$

\section{Step 2: Calculate the similarity}

The cosine similarity is applied to computed a similarity score between $V_{1}$ and $V_{2}$.

$$
\operatorname{sim}\left(S_{1}, S_{2}\right)=\cos \left(V_{1}, V_{2}\right)=0.78
$$

We note that the similarity result between the two sentences is better than the previous method.

\subsubsection{Part-of-speech weighting Method}

An alternative technique is the application of the Part-of-Speech tagging (POS tag) for identification of words that are highly descriptive in each input sentence (Lioma and Blanco, 2009). For this purpose, we have used the POS tagger for Arabic language proposed by G. Braham et al. (2012) to estimate the part-of-speech of each word in sentence. Then, a weight is assigned for each type of tag in the sentence. For example, ver $b=0.4$, noun $=0.5$, adjective $=0.3$, preposition $=$ 0.1 , etc.

The similarity between $S_{1}$ and $S_{2}$ is obtained in three steps (Ferrero et al., 2017) as follows:

\section{Step 1: POS tagging}

In this step the POS tagger of G. Braham et al. (2012) is used to estimate the POS of each word in sentence.

$$
\left\{\begin{array}{l}
\text { Pos_tag }\left(S_{1}\right)=\operatorname{Pos}_{w_{1}}, \operatorname{Pos}_{w_{2}}, \ldots, \text { Pos }_{w_{i}} \\
\operatorname{Pos} \_t a g\left(S_{2}\right)=\operatorname{Pos}_{w_{1}^{\prime}}, \operatorname{Pos}_{w_{2}^{\prime}}, \ldots, \operatorname{Pos}_{w_{j}^{\prime}}
\end{array}\right.
$$

The function Pos_tag $\left(S_{i}\right)$ returns for each word $w_{k}$ in $S_{i}$ its estimated part of speech $\operatorname{Pos}_{w_{k}}$.

\section{Step 2: POS weighting}

At this point we should mention that, the weight of each part of speech can be fixed empirically. Indeed, we based on the training data of SemEval2017 (Task 1) $)^{3}$ to fix the POS weights.

$$
\left\{\begin{array}{l}
V_{1}=\sum_{k=1}^{i} \text { Pos_weight }\left(\text { Pos }_{w_{k}}\right) * v_{k} \\
V_{2}=\sum_{k=1}^{j} \text { Pos_weight }\left(\text { Pos }_{w_{k}^{\prime}}\right) * v_{k}^{\prime}
\end{array}\right.
$$

where Pos_weight $\left(\operatorname{Pos}_{w_{k}}\right)$ is the function which return the weight of POS tagging of $w_{k}$.

\section{Step 3: Calculate the similarity}

Finally, the similarity between $S_{1}$ and $S_{2}$ is obtained by calculating the cosine similarity between $V_{1}$ and $V_{2}$ as follows: $\operatorname{sim}\left(S_{1}, S_{2}\right)=\cos \left(V_{1}, V_{2}\right)$.

\footnotetext{
${ }^{3}$ http://alt.qcri.org/semeval2017/task1/data/uploads/
} 


\section{Example:}

Let us continue with the same example, and suppose that POS weights are:

\begin{tabular}{c|c|c|c|c} 
verb & noun & noun_prop & adj & prep \\
0.4 & 0.5 & 0.7 & 0.3 & 0.1
\end{tabular}

\section{Step 1: Pos tagging}

The function Pos_tag $\left(S_{i}\right)$ is applied to each sentence.

$$
\left\{\begin{array}{l}
\text { Pos_tag }\left(S_{1}\right)=\text { verb noun_prop noun } \\
\text { Pos_tag }\left(S_{2}\right)=\text { noun_prop verb adj noun }
\end{array}\right.
$$

Step 2: Sum of vectors with POS weighting

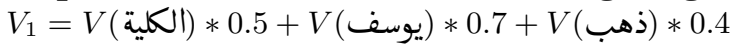

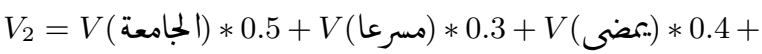
$V($ ) 0.7

\section{Step 3: Calculate the similarity}

$$
\operatorname{sim}\left(S_{1}, S_{2}\right)=\cos \left(V_{1}, V_{2}\right)=0.82
$$

\subsubsection{Mixed weighting}

We have proposed another method (after the competition), this method propose to use both IDF and the POS weightings simultaneously. The similarity between $S_{1}$ and $S_{2}$ is obtained as follows:

$$
\left\{\begin{array}{l}
V_{1}=\sum_{k=1}^{i} i d f\left(w_{k}\right) * \text { Pos_weight }\left(\text { Pos }_{w_{k}}\right) * v_{k} \\
V_{2}=\sum_{k=1}^{j} i d f\left(w_{k}^{\prime}\right) * \text { Pos_weight }\left(\text { Pos }_{w_{k}^{\prime}}\right) * v_{k}^{\prime}
\end{array}\right.
$$

If we apply this method to the previous example, using the same weights in Section 3.2 and 3.3, we will have: $\operatorname{Sim}\left(S_{1}, S_{2}\right)=\operatorname{Cos}\left(V_{1}, V_{2}\right)=0,87$.

\section{Experiments And Results}

\subsection{Preprocessing}

In order to normalize the sentences for the semantic similarity step, a set of preprocessing are performed on the data set. All sentences went through by the following steps:

1. Remove Stop-word, punctuation marks, diacritics and non letters.

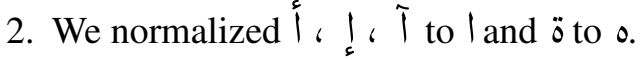

3. Replace final $\checkmark$ followed by $s$ with

4. Normalizing numerical digits to Num.

\subsection{Tests and Results}

To evaluate the performance of our system, our four approaches were assessed based on their accuracy on the 250 sentences in the STS 2017 Monolingual Arabic Evaluation Sets v1.1 ${ }^{4}$. We calculate the Pearson correlation between our assigned semantic similarity scores and human judgements. The results are presented in Table 1.

${ }^{4}$ http://alt.qcri.org/semeval2017/task1/data/uploads /sts2017.eval.v1.1.zip

\begin{tabular}{c|c}
\hline Approach & Correlation \\
\hline Basic method (run 1) & 0.5957 \\
IDF-weighting method (run 2) & 0.7309 \\
POS tagging method (run 3) & 0.7463 \\
Mixed method & 0.7667 \\
\hline
\end{tabular}

Table 1: Correlation results

These results indicate that when the no weighting method is used the correlation rate reached 59.57\%. Both IDF-weighting and POS tagging approaches significantly outperformed the correlation to more than $73 \%$ (respectively $73.09 \%$ and $74.63 \%$ ). We noted that, the Mixed method achieve the best correlation (76.67\%) of the different techniques involved in the Arabic monolingual pairs STS task.

\section{Conclusion and Future Work}

In this article, we presented an innovative word embedding-based system to measure semantic relations between Arabic sentences. This system is based on the semantic properties of words included in the word-embedding model. In order to make further progress in the analysis of the semantic sentence similarity, this article showed how the IDF weighting and Part-of-Speech tagging are used to support the identification of words that are highly descriptive in each sentence. In the experiments we have shown how these techniques improve the correlation results. The performance of our proposed system was confirmed through the Pearson correlation between our assigned semantic similarity scores and human judgements. As future work, we are going to combine these methods with those of other classical techniques in NLP field such as: n-gram, fingerprint and linguistic resources.

\section{Acknowledgments}

This work was carried out as part of a PNE scholarship funded by Ministry of Higher Education and Scientific Research of Algeria, with an international collaboration between two research laboratories: LIM Laboratoire d'Informatique et de Mathmatiques Laghouat, Algeria and LIG Laboratoire d'Informatique de Grenoble (GETALP Team), France. 


\section{References}

Eneko Agirre, Carmen Baneab, Claire Cardiec, Daniel Cerd, Mona Diabe, Aitor Gonzalez-Agirrea, Weiwei Guof, Inigo Lopez-Gazpioa, Montse Maritxalara, Rada Mihalceab, et al. 2015. Semeval-2015 task 2: Semantic textual similarity, english, spanish and pilot on interpretability. In Proceedings of the 9th international workshop on semantic evaluation (SemEval 2015), pages 252-263.

Yu Chen and Andreas Eisele. 2012. Multiun v2: Un documents with multilingual alignments. In LREC, pages 2500-2504.

Ronan Collobert and Jason Weston. 2008. A unified architecture for natural language processing: Deep neural networks with multitask learning. In Proceedings of the 25th international conference on Machine learning, pages 160-167. ACM.

Jérémy Ferrero, Frédéric Agnès, Laurent Besacier, and Didier Schwab. 2017. Using Word Embedding for Cross-Language Plagiarism Detection. In European Association for Computational Linguistics (EACL), Volume "short papers" EACL 2017, Valence, Spain, April.

ksucorpus. 2012. King saud university corpus, http://ksucorpus.ksu.edu.sa/ar/ (accessed january 20,2017$)$.

Christina Lioma and Roi Blanco. 2009. Part of speech based term weighting for information retrieval. In European Conference on Information Retrieval, pages 412-423. Springer.

Meedan. 2012. Meedan's open source arabic english, https://github.com/anastaw/meedan-memory, (accessed january 20,2017).

Tomas Mikolov, Kai Chen, Greg Corrado, and Jeffrey Dean. 2013a. Efficient estimation of word representations in vector space. In In: ICLR: Proceeding of the International Conference on Learning Representations Workshop Track, pages 1301-3781.

Tomas Mikolov, Ilya Sutskever, Kai Chen, Greg S Corrado, and Jeff Dean. 2013b. Distributed representations of words and phrases and their compositionality. In Advances in neural information processing systems, pages 3111-3119.

Tomas Mikolov, Wen-tau Yih, and Geoffrey Zweig. 2013c. Linguistic regularities in continuous space word representations. In Hlt-naacl, volume 13, pages 746-751.

Andriy Mnih and Geoffrey E Hinton. 2009. A scalable hierarchical distributed language model. In D. Koller, D. Schuurmans, Y. Bengio, and L. Bottou, editors, Advances in Neural Information Processing Systems 21, pages 1081-1088. Curran Associates, Inc.
Hazem M Raafat, Mohamed A Zahran, and Mohsen Rashwan. 2013. Arabase-a database combining different arabic resources with lexical and semantic information. In $K D I R / K M I S$, pages 233-240.

Motaz K Saad and Wesam Ashour. 2010. Osac: Open source arabic corpora. In 6th ArchEng Int. Symposiums, EEECS, volume 10.

Gerard Salton and Christopher Buckley. 1988. Termweighting approaches in automatic text retrieval. Information processing \& management, 24(5):513523.

Didier Schwab. 2005. Approche hybride-lexicale et thématique-pour la modélisation, la détection et lexploitation des fonctions lexicales en vue de lanalyse sémantique de texte. Ph.D. thesis, Université Montpellier II.

Jörg Tiedemann. 2012. Parallel data, tools and interfaces in opus. In $L R E C$, volume 2012, pages 2214 2218.

Joseph Turian, Lev Ratinov, and Yoshua Bengio. 2010. Word representations: a simple and general method for semi-supervised learning. In Proceedings of the 48th annual meeting of the association for computational linguistics, pages 384-394. Association for Computational Linguistics.

WikiAr. 2006. Arabic wikipedia corpus, http://linguatools.org/tools/corpora/wikipediamonolingual-corpora/, (accessed january 21,2017). 